\title{
Analysis of missed diagnosis of orbital foreign bodies
}

\author{
LEI ZHOU, SU-YAN LI, JIAN-PING CUI, ZHI-YANG ZHANG and LI-NA GUAN \\ Research Institute of Eye Diseases, Xuzhou, Jiangsu 221002, P.R. China
}

Received February 9, 2016; Accepted November 8, 2016

DOI: $10.3892 /$ etm.2017.4146

\begin{abstract}
A clinical analysis of diagnosis was performed as well as the management of orbital foreign bodies, to investigate the methods to avoid missed diagnosis. A total of 15 cases of an orbital foreign body was reviewed, and for these cases, the clinical manifestation, imaging data and operative situation were studied. Among the patients, 4 cases turned out to have wooden, 3 metallic, 2 glass, 2 bones, and 4 other foreign bodies. Twelve cases had received debridement and suture before our management, and 1 foreign body was treated more than once. In conclusion, detailed traumatic history and imaging examination are necessary for the diagnosis of orbital foreign bodies, while prompt diagnosis, accurate location and professional surgical skills are important for the treatment.
\end{abstract}

\section{Introduction}

Orbital foreign body mainly enters the orbit between the eye and orbital wall, but may occasionally enter the orbit through the eye or by the paranasal sinus into the orbit. According to the foreign species, they can be divided into metallic bodies and non-metallic ones, and the latter can be further divided into irritant and non-irritant foreign body. Stimulation of a foreign body is an unstable substance of physicochemical properties, such as plant bodies, gunpowder, grease and wax, which can cause severe tissue reaction or infection and inflammation. Non-irritating common foreign body such as glass, stones, gravel, and plastic do not cause severe complications afterwards except mechanical injury $(1,2)$.

In this study, a retrospective analysis of 15 patients with an orbital foreign body was performed at the Research Institute of Eye Diseases from May 2007 to November 2011. Among these, there were 4 cases of plant bodies, 3 cases of metallic foreign body, 2 cases of glass foreign body, 2 cases of osseous foreign body and 4 others. Twelve cases had debridement and suture before removing the foreign bodies. In 1 case, plant bodies were taken out in two separate operations. The shortest miss diagnosis time was 3 days, and the longest was

Correspondence to: Dr Lei Zhou, Research Institute of Eye Diseases, 6 Fu Guo Street, Xuzhou, Jiangsu 221002, P.R. China E-mail: xzzljs@sina.com

Key words: orbital foreign body, diagnosis, ocular trauma
15 months. According to the clinical imaging data and each operation case, the reasons for the easily missed diagnosis of orbital foreign body were analyzed. This study was approved by the Ethics Committee of Research Institute of Eye Diseases. Signed written informed consents were obtained from all participants before the study.

\section{Case report}

Case 1, subcutaneous eyewinker. A 25-year-old female patient had an unknown object stab wound, and a suture skin wound by local debridement suture, asked for treatment because of subcutaneous eyewinker feeling. The patient was examined for left lacrimal sac area accessible cords, hard nodules, wound healing, and flushing lacrimal passage. Ophthalmology examination had no positive signs. CT examination was conducted to examine the lacrimal sac area with a bar-shaped high-density shadow, and suspected subcutaneous eyewinker. Under local anesthesia, the area along the original wound was expanded and several pieces of tin aluminum soft foreign body were removed (Fig. 1).

Case 2, metal foreign body. A male patient, aged 52 years, had reinforced stab wound with the local skin suture, and had postoperative ptosis and eye movement disorder. CT examination showed that the metallic foreign body was present in orbit above. Under general anesthesia, along the original road, a metal foreign body, approximately $3 \mathrm{~cm}$ in length was removed (Fig. 2).

Case 3, wooden foreign body. A male patient aged 32 years had no special treatment after a drunken motorcycle accident. A week later, an upper eyelid mass was found at the admitting hospital where the patient was given antibiotic treatment and the mass was considered a 'sty' with poor efficacy. In December 2010 the patient presented at the Research Institute of Eye Diseases, where a CT scan showed that there were 2 pieces of low-density shadows above orbital with suspected foreign body. Under local anesthesia, the region was explored, and 2 blocks of wooden foreign body were removed.

Case 4, wooden foreign body. A male patient, aged 56 years, had a branch stab wound. Three months after debridement and suturing, postoperative wound repeated inflammation with secreta outflow and eyeball movement disorder to the internal oblique fixed, with declining eyesight. In the local 


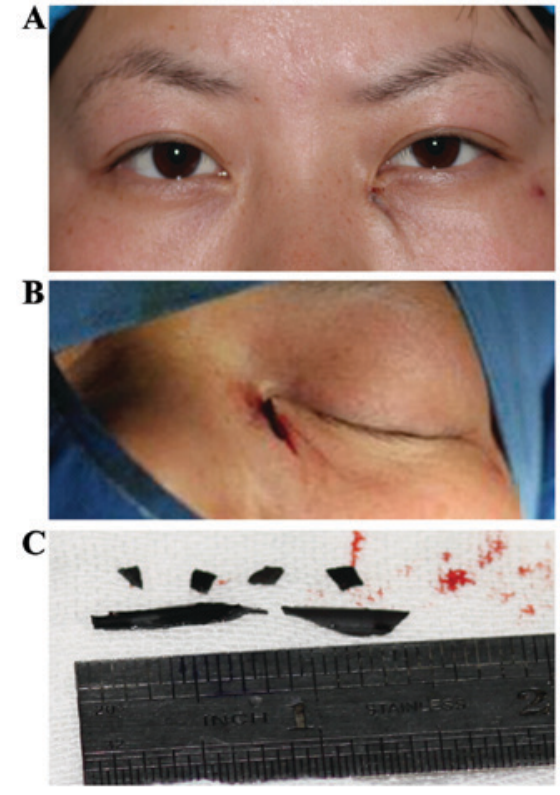

Figure 1. Clinical data of case 1. (A) Subcutaneous eyewinker in the left eye lacrimal sac area, (B) skin incision along the original wound, and (C) tin aluminum foreign body.
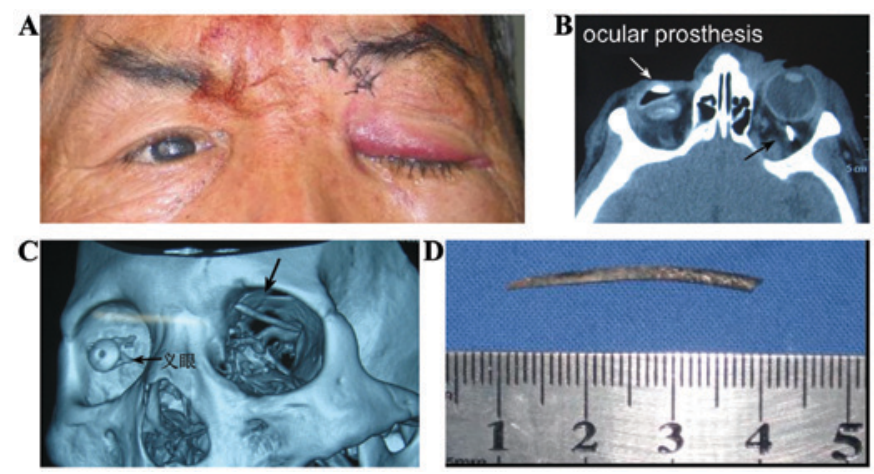

Figure 2. Clinical data of case 2. (A) The left eye ptosis after debridement and suturing. (B and C) CT plain scan, 3D reconstruction showed high-density shadow of orbital foreign body. (D) After taking out the metal foreign body.
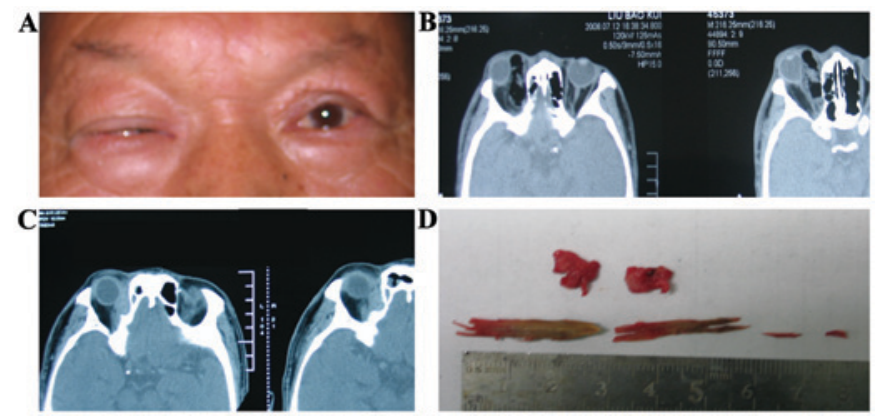

Figure 3. Clinical data of case 4. (A) Ocular dyscinesia of the right eye. (B and C) CT plain scan showed irregular high-density shadows of orbital foreign body; (D) after taking out the wooden foreign body.

hospitals no foreign body was identified by $\mathrm{CT}$ check. In our hospital, the CT was rechecked and an irregular high-density shadow was identified in the medial orbital with a low-density intermediate shadow, and intraorbital foreign body was suspected. Under general anesthesia exploratory operation was carried out and several short wooden foreign bodies were removed (Fig. 3).

Case 5, chemical foreign bodies. Left upper eyelid of a 5 -year-old boy was injured by a door. A week with repeated redness and free remission after antibiotic treatment. In our hospital, CT scan analysis identified irregular high-density shadow above the orbital, a shadow similar to a vein stone in the middle. His examination history was checked, 3 months earlier, debridement and suturing was performed following a crayon stab wound. Under general anesthesia, local proliferation of granulation tissue wrapped with green wax foreign body was identified (Fig. 4).

Case 6, glass foreign body. A male patient, aged 3 years, touched the door after being hurt by glass. The local hospital took skin wound debridement and sutured the superciliary arch. Due to eyeball movement disorder and poor eyelid closure, treatment was requested. In our hospital, the CT scan revealed a high-density foreign body shadow bar under orbital. The glass foreign body was removed under general anesthesia (Fig. 5).

Case 7, bone foreign body. A male patient aged 56 years, after falling, had diplopia with right-eye proptosis. The CT scan showed a fracture of right lateral orbital zygomatic arch with intraorbital foreign body and lateral rectus muscle transposition. Bone foreign body was removed under general anesthesia (Fig. 6).

\section{Discussion}

The diagnosis of orbital foreign body has the following limitations: i) The complexity of trauma among children, and intoxicated patients who cannot describe detailed trauma after a car accident, explosion, or the moment of injury and also cannot determine the injury objects. ii) The imaging findings of diversification, especially some non-metal foreign body lack typical imaging findings. iii) During the clinical diagnosis and treatment process, doctors often only pay attention to the wound treatment, but fail to make an early diagnosis of orbital foreign body resulting in missed diagnosis. Ophthalmology doctors should master data, detailed history of skilled imaging data, be familiar with orbital operation approach and have operation skills, in order to reduce the rate of missed diagnosis of orbital foreign body, and to improve the success rate of removal of foreign bodies $(1,2)$. Therefore, we summarize the experience of diagnosis and treatment of orbital foreign bodies as follows:

i) Eyes perforating injury needs to pay attention to the possibility of intraorbital foreign body retention: All orbital penetrating injury patients should be asked in detail about the environment where injury or wound occurred and possible vulnerant. For local repeated inflammation, unknown cause should be traced back to the history. The results of debridement and suturing should be examined properly and attention should be paid for the possible presence of residual foreign body. Once there are visible symptoms, such as pain, swelling, heat, eyeball movement disorder, proptosis, eyelid closure, 

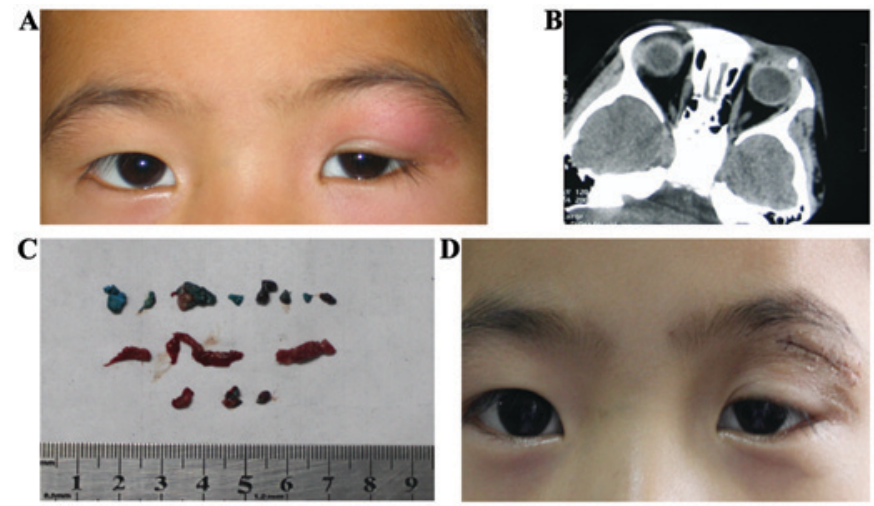

Figure 4. Clinical data of case 5. (A) The child asks for treatment repeatedly for the left upper eyelid swelling; (B) CT showed subcutaneous irregular high-density shadow; (C) subcutaneous waxy foreign body; (D) appearance 1 week after operation.
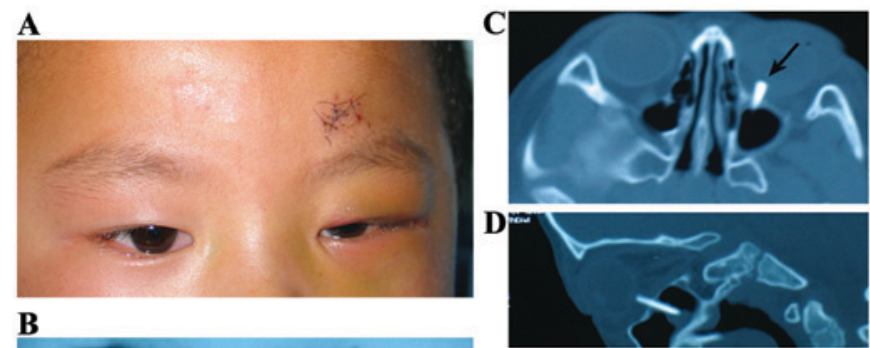

B
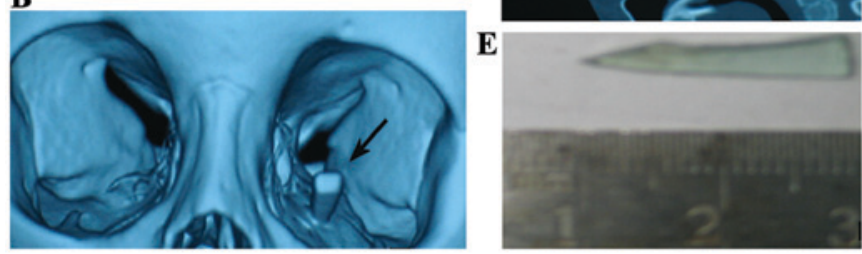

Figure 5. Clinical data of case 6. (A) Preoperative appearances; (B) CT reconstruction showed the foreign bodies in the inferior wall of orbit; (C and D) horizontal, sagittal CT images; (E) glass foreign body.

and eyesight decline, further consideration should be given to the possibility of orbital cellulitis and orbital apex syndrome, orbital fracture, nerve injury, optic nerve injury, carotid cavernous fistula, and combined with the history, signs, imaging effects, an assessment of injury should be made to further eliminate the intraorbital foreign body.

ii) Improved imaging examination: CT examination is a reliable basis for diagnosis of orbital foreign body. Ophthalmologists should be familiar with the CT imaging of the intraorbital foreign body with different nature. CT check generally chooses axial and coronal to fully understand the nature and the position of the foreign body, and avoid some small foreign bodies on the orbital roof and orbital floor resulting in misdiagnosis caused by the volume effect. Tissue window can display the fracture and rectus damage situation of orbital. Metal foreign body is easy to be found in the CT examination. When it is a metallic foreign body, the artifacts can be eliminated by using bone window. Glass foreign body performance is that the density value is between $+300--+600 \mathrm{Hu}$ and the boundary is clear. Plant or wooden foreign bodies in the early period in general are negative on $\mathrm{CT}$, but could be identified with hemorrhage and air. Long-term retention of plant foreign bodies due to absorption
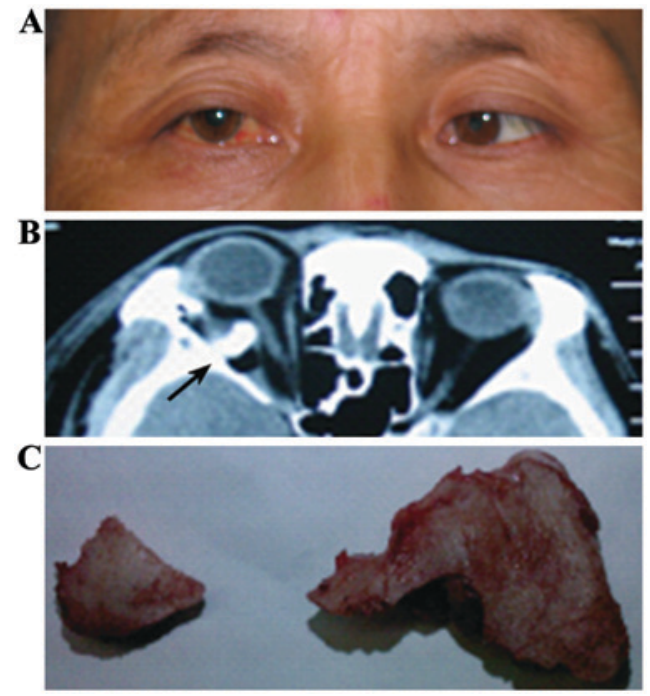

Figure 6. Clinical data of case 7. (A) Right eye proptosis, in turn limited; (B) CT showed the right orbital bone density shadow; (C) postoperative removal of bone foreign body.

of tissue fluid or chronic inflammation and other factors can be manifested as irregular high-density shadow (3). Bone foreign body in general is the same as defect orbital bone with the same density.

iii) Indications and principle of orbital foreign body removal: a) All stimulation of foreign body such as plants, non-metal foreign body should be completely removed by operation as soon as possible, in order to avoid the pyogenic infection and fistula formation. b) A foreign body of eye movement disorder, compression symptoms and visual dysfunction should be treated. c) Orbital small metallic foreign body, glass and sand foreign bodies that have not caused any eye symptoms can be observed without removal. (d) For greater operation difficulty and vulnerable injury of the more important normal tissue, blood vessels, nerves and other factors, foreign body near the orbital apex, follow-up observation is preferred, as long as it does not cause significant visual impairment (3-6).

iv) Operation method to take out orbital foreign body: a) A general anesthesia is usually a good idea to eliminate the stress of the patient that will be good to cooperate, so surgeons can fully expose the operation field and have full debridement. If the object is clear with the superficial location, local anesthesia can also be used. b) Generally, operation incision along the original wound or foreign body can be near the skin incision, when necessary, it is appropriate to expand the incision for correct use of instruments. c) Along the original road into looking for foreign body, Meilan mark can be injected through road before entering. d) During separation, it was found that the pus cavity had reached the peripheral location of foreign body. e) Foreign body should be removed and area around the abscess cavity should be cleaned up, because plant bodies could contaminate during the process of removal and break foreign body into several segments or debris which should be cleaned as far as possible. Foreign body that has caused chemical reaction and contamination or inflammatory reactions leading to decaying of tissue, should be appropriately removed and rinsed thoroughly $(7,8)$. 
With the development of transportation and city construction, orbital trauma incidences are increasing every year. In addition, since the reforms and opening up, great changes have taken place in China's occupational structure, but grassroots employees such as the production workers and agricultural laborers still account for a large proportion. But the basic labor people especially manual workers are at a high risk of ocular trauma and multiple injuries, and soon after injuries, majority of them have treatment in primary hospitals because of distance and time. So ophthalmologists particularly primary ophthalmologists should be fully aware of more complexities of diagnosis and treatment of orbital foreign body in order to avoid misdiagnosis, missed diagnosis and delay in treatment. In the process of diagnosis and treatment, attention should be paid to the following problems: i) Inquire the details of injuries, and be alert to the possibility of intraorbital foreign bodies. ii) During the emergency treatment of the wound, necessary cleaning and exploration should be performed. iii) After the injury or wound suture, CT check is necessary. iv) Ability to correctly read scans and proper clinical training. v) For patients with ocular symptoms or visual dysfunction, the diagnosis should be further clarified, examined and explored. vi) Patients who fail to be clearly diagnosed, should be transferred to the orbital disease specialists or higher level hospitals for further treatment as soon as possible.

\section{References}

1. Elsner H, Hoerauf $\mathrm{H}$ and Laqua $\mathrm{H}$ : Glass orbital foreign body 15 years after windshield injury. Ophthalmologe 99: 488-489, 2002 (In German).

2. Tyranski Nielsen R, Christensen S, Buur Rosbach S and Bjerre PK: Orbital injury following accidental fall. Intra-orbital wooden foreign body. Radiologe 38: 545-547, 1998 (In German).

3. Zhu Y, Li ZG and Zhang XF: Problems in diagnosis and treatment of orbital foreign body - an analysis of 24 cases. Zhonghua Yan Ke Za Zhi 44: 676-680, 2008 (In Chinese).

4. Chung IY, Seo SW, Han YS, Kim E and Jung JM: Penetrating retrobulbar orbital foreign body: A transcranial approach. Yonsei Med J 48: 328-330, 2007

5. Naik MN, Das S, Oluyemi F and Honavar SG: An extraordinary orbital foreign body. Ophthal Plast Reconstr Surg 27: e149-e152, 2011.

6. Zinreich SJ, Miller NR, Aguayo JB, Quinn C, Hadfield R and Rosenbaum AE: Computed tomographic three-dimensional localization and compositional evaluation of intraocular and orbital foreign bodies. Arch Ophthalmol 104: 1477-1482, 1986.

7. Neumann K, Ehrich D and Bloching M: Orbital foreign bodies - diagnostics, therapy and management. Laryngorhinootologie 84: 187-192, 2005.

8. van Putten SM, Wübben M, Plantinga JA, Hennink WE, van Luyn MJ and Harmsen MC: Endotoxin contamination delays the foreign body reaction. J Biomed Mater Res A 98: 527-534, 2011. 